\title{
Transcriptional Activator GmrA, Encoded in Genomic Island OI-29, Controls the Motility of Enterohemorrhagic Escherichia coli 0157:H7
}

\author{
Bin Yang 1,2,3, Shaomeng Wang 1,2, Jianxiao Huang 1,2, Zhiqiu Yin 1,2, Lingyan Jiang 1,2,3, \\ Wenqi Hou ${ }^{1,2}$, Xiaomin $\mathrm{Li}^{1,2}$ and Lu Feng ${ }^{1,2,3,4,5 *}$ \\ ${ }^{1}$ TEDA Institute of Biological Sciences and Biotechnology, Nankai University, Tianjin, China, ${ }^{2}$ Key Laboratory of Molecular \\ Microbiology and Technology, Ministry of Education, Tianjin, China, ${ }^{3}$ Tianjin Key Laboratory of Microbial Functional \\ Genomics, Tianjin, China, ${ }^{4}$ State Key Laboratory of Medicinal Chemical Biology, Nankai University, Tianjin, China, \\ ${ }_{5}^{5}$ SynBio Research Platform, Collaborative Innovation Center of Chemical Science and Engineering (Tianjin), Tianjin, China
}

OPEN ACCESS

Edited by:

Dongsheng Zhou,

Beijing Institute of Microbiology

and Epidemiology, China

Reviewed by:

Grzegorz Wegrzyn

University of Gdańsk, Poland

Miguel A. De la Cruz,

IMSS, Mexico

Antonio Juárez,

Universitat de Barcelona, Spain

Roy Michael Robins-Browne,

University of Melbourne, Australia

*Correspondence:

Lu Feng

fenglu63@nankai.edu.cn

Specialty section: This article was submitted to

Infectious Diseases,

a section of the journal

Frontiers in Microbiology

Received: 13 October 2017 Accepted: 12 February 2018

Published: 22 February 2018

Citation: Yang B, Wang S, Huang J, Yin Z, Jiang $L$, Hou W, Li $X$ and Feng $L$ (2018) Transcriptional Activator GmrA, Encoded in Genomic Island Ol-29, Controls the Motility of Enterohemorrhagic Escherichia coli 0157:H7. Front. Microbiol. 9:338. doi: 10.3389/fmicb.2018.00338
Enterohemorrhagic Escherichia coli $\mathrm{O} 157: \mathrm{H7}$ is a major human enteric pathogen capable of causing large outbreaks of severe infections that induce bloody diarrhea, hemorrhagic colitis, and hemolytic uremic syndrome. Its genome contains 177 unique $\mathrm{O}$ islands (Ols) including those carrying the main virulence elements, Shiga toxin-converting phages (Ol-45 and Ol-93) and locus for enterocyte effacement (OI148). However, many of these islands harbor only genes of unknown function. Here, we demonstrate that OI-29 encodes a newly discovered transcriptional activator, Z0639 (named GmrA), that is required for motility and flagellar synthesis in 0157:H7. GmrA directly binds to the promoter of fliA, an RNA polymerase sigma factor, and thereby regulates flagellar genes controlled by FliA. Expression of gmrA is maximal under host conditions $\left(37^{\circ} \mathrm{C}\right.$, neutral $\mathrm{pH}$, and physiological osmolarity), and in the presence of host epithelial cells, indicative of a role of this gene in infection by promoting motility. Finally, GmrA was found to be a widespread regulator of bacterial motility and flagellar synthesis in different pathotypes of $E$. coli. Our work largely enriches our understanding of bacterial motility control, and provides another example of regulators acquired laterally that mediate flagellar synthesis.

Keywords: enterohemorrhagic Escherichia coli, O island 29, Z0639, motility, flagellar synthesis

\section{INTRODUCTION}

Enterohemorrhagic Escherichia coli (EHEC) is a principally foodborne pathogen linked to serious diseases, including hemorrhagic colitis and hemolytic uremic syndrome (Monteiro et al., 2016). Adult cattle and other farm animals are the main reservoirs of many EHEC serotypes (Caprioli et al., 2005), and humans become infected via contaminated food and water (Watanabe, 2012; Monteiro et al., 2016). The major virulence determinants of EHEC include the ability to produce Shiga toxins, adhere to host epithelial cells, and form histopathological attaching and effacing lesions (Watanabe, 2012). Of which, the induction of such lesions is most critical for the establishment of successful colonization to cause infection, and this ability is conferred by locus of 
enterocyte effacement (LEE), which consists of five polycistronic operons (LEE1 to LEE5) encoding a type III secretion system and associated effectors (Wong et al., 2011; Monteiro et al., 2016). Gene expression from this locus is regulated via a complex mechanism to ensure the expression occurs only under host conditions (Mellies et al., 2007; Connolly et al., 2015). While LEE1-encoded ler is the master activator of all LEE operons, a range of global and specific regulators are also involved, such as H-NS, IHF, QseA, GrvA, GadE, Pch, EivF, EtrA, and Hha (Connolly et al., 2015).

Bacterial motility is often considered to play diverse roles in pathogenesis, including in migration to an optimal site in the host, colonization or invasion, survival at the infection site, and post-infection dispersal (Chaban et al., 2015). One of the most widespread motility machines in bacteria is the flagellum, a complex macromolecular structure driven by a motor which rotates a long, curved filament extending from the cell envelope (Berg, 2003; Chaban et al., 2015). A flagellum consists of a basal body (rotary motor), a hook (universal joint), and a filament (propeller) formed through polymerization of flagellin (FliC) (Erhardt et al., 2010). More than 50 genes are required to form and operate the flagellum, the expression of which is stringently controlled to minimize unnecessary energy expenditure. The regulation of flagellar gene expression is highly complex, with FlhD and FlhC as master transcriptional activators (Claret and Hughes, 2000). These proteins form an $\mathrm{FlhD}_{4} \mathrm{C}_{2}$ complex and boost expression of flagellar genes, both directly and via FliA, an RNA polymerase sigma factor (Claret and Hughes, 2000; Chevance and Hughes, 2008). Flagellar synthesis is also regulated by various proteins and sRNAs, including MatA, CRP, H-NS, HdfR, QseBC, and DksA, which regulate $f$ lhDC transcriptionally, and thereby control fliA and other flagellar genes (McCarter, 2006; Duan et al., 2013), and H-NS, CsgD, and NsrR, that control flagella-based motility by modulating fliA transcription (McCarter, 2006; Duan et al., 2013).

Escherichia coli O157:H7, the most well-known EHEC strain, is also the most common serotype associated with large infection outbreaks (Bavaro, 2012; Ho et al., 2013). Notably, the O157:H7 genome contains $177 \mathrm{O}$ islands (OI) that are not present in non-pathogenic E. coli K-12 (Perna et al., 2001). These islands comprise the main known virulence elements in $\mathrm{O} 157: \mathrm{H} 7$, and include LEE (OI-148) and Shiga toxin-converting phages (OI-45 and OI-93) (Hayashi et al., 2001; Perna et al., 2001). The functions of several other islands were also established in recent years, revealing more virulence factors associated with adherence and motility. OI-1 was found to encode a repressor of flagellar synthesis and bacterial motility, while OI172 was determined to encode an activator (Allison et al., 2012; Xu et al., 2013). OI-15 is now known to encode an AIDA-like adhesin required for adherence in vitro and in vivo (Yin et al., 2009a), while OI-48 was determined to encode tellurite resistance, Iha, and urease to promote adherence to the host intestinal epithelium (Yin et al., 2009b). OI-50 and OI-51 encode virulence regulators and other effectors required for infection (Tree et al., 2011; Flockhart et al., 2012), while OI-71 encodes NleA, a type III secretion system effector encoded outside of LEE (Gruenheid et al., 2004). OI122 also carries the virulence genes efal/lifA, which encode adherence/lymphocyte inhibitory factor and is required for pathogen adhesion in vitro and suppression of the host immune response (Karmali et al., 2003). Nevertheless, most genes in $\mathrm{O}$ islands have not been characterized and are of unknown function.

We now demonstrate that Z0639, renamed as GmrA (Genomic island-encoded Motility Regulator $\underline{\mathrm{A}}$ ), encoded in OI-29 is a newly discovered transcriptional activator that regulates flagellar synthesis and motility in E. coli O157:H7. GmrA directly binds to the promoter of fliA based on EMSA and ChIP-qPCR analysis, and thereby regulates flagellar genes controlled by FliA. gmrA expression is maximal at $37^{\circ} \mathrm{C}$, neutral $\mathrm{pH}$, physiological osmolarity, and in the presence of host epithelial cells. Finally, GmrA was found to be a widespread regulator of bacterial motility in pathogenic E. coli. This work reveals a new example of regulators acquired laterally for the control of flagella synthesis.

\section{MATERIALS AND METHODS}

\section{Bacterial Strains, Plasmids, and Culture}

Bacterial strains and plasmids are summarized in Supplementary Table S1. Mutant strains were generated by the $\lambda$ Red recombinase system (Datsenko and Wanner, 2000; Murphy and Campellone, 2003), and verified by PCR and sequencing. Complemented strains were constructed by cloning the appropriate genes into low-copy plasmid pACYC184, and then by electroporating the resulting constructs into the corresponding mutants. Strains for protein purification were constructed by cloning genes of interest into the pET28a expression vector, and then by electroporating the resulting constructs into E. coli BL21. All genetic manipulation on virulent bacterial strains was performed according to standard biosecurity and institutional safety procedures. Primers for all manipulations are listed in Supplementary Table S2. Unless otherwise specified, all strains were grown in Luria-Bertani (LB) broth supplemented as needed with $100 \mu \mathrm{g} \mathrm{mL}^{-1}$ ampicillin, $15 \mu \mathrm{g} \mathrm{mL}^{-1}$ chloramphenicol, and $50 \mu \mathrm{g} \mathrm{mL}^{-1}$ kanamycin.

\section{Bacterial Adherence}

Adherence was assayed as previously described (Dibb-Fuller et al., 2001). Briefly, HeLa and Caco-2 cells, obtained from Shanghai Institute of Biochemistry and Cell Biology, Chinese Academy of Sciences (Shanghai, China), were grown at $37^{\circ} \mathrm{C}$ in $5 \% \mathrm{CO}_{2}$ until confluent, washed three times with pre-warmed PBS, and the medium was replaced with fresh DMEM without antibiotics and fetal bovine serum. Cells were then infected with bacterial cultures in exponential phase $\left(10^{8} \mathrm{CFU} /\right.$ well $)$. After $3 \mathrm{~h}$, unattached bacteria were removed by washing the wells six times with PBS. Cells were then lysed with $0.1 \%$ SDS, and resulting lysates were serially diluted and plated on LB agar. Attachment efficiency was calculated as the numbers of adherent bacteria per cell. 


\section{Quantitative Real-Time PCR (qRT-PCR)}

Total RNA was prepared using TRIzol ${ }^{\circledR}$ LS Reagent (Invitrogen: 15596018) following the manufacturer's instructions, and digested with RNase-Free DNase I (Qiagen: 79254) to eliminate contaminating genomic DNA. First-strand cDNA was synthesized using PrimeScript $1^{\text {st }}$ Strand cDNA Synthesis Kit (Takara: D6110A), and analyzed by qRT-PCR on an ABI 7500 system (Applied Biosystems) using SYBR Green PCR master mix (Applied Biosystems: 4367659). The $16 \mathrm{~S}$ rRNA gene $r r s H$ was used as reference, and relative differences in gene expression were calculated by the cycle threshold method $\left(2^{-\Delta \Delta c t}\right.$ ) (Livak and Schmittgen, 2001; Tasara and Stephan, 2007). Data were collected from at least three biological replicates.

\section{Motility}

Overnight cultures were adjusted to optical density 1.0 at $600 \mathrm{~nm}$, of which $1 \mu \mathrm{L}$ was then stab-inoculated using a sterile pipette tip into $0.25 \% \mathrm{LB}$-agar plates supplemented with ampicillin as needed. Agar plates were then incubated at 30 or $37^{\circ} \mathrm{C}$ for $10 \mathrm{~h}$, at which point the diameter of the swimming zone around the inoculation site was measured. All strains were tested in triplicate, and each experiment was carried out on three separate occasions.

\section{Transmission Electron Microscopy}

Strains were cultured in LB broth at $37^{\circ} \mathrm{C}$ until optical density 0.8 at $600 \mathrm{~nm}$. Samples $(1 \mathrm{~mL})$ were then harvested at 2,000 rpm for $5 \mathrm{~min}$, resuspended in an equal volume of distilled water, of which $10 \mu \mathrm{L}$ was dropped and adsorbed for $3 \mathrm{~min}$ to carbon-stabilized Formvar supports on 200-mesh copper grids. Cells were then stained by submerging the grids for $3 \mathrm{~min}$ in $2 \% \mathrm{wt} / \mathrm{vol}$ sodium phosphotungstate, and imaged on a HITACHI HT7700 transmission electron microscope operating at $100 \mathrm{kV}$ and fitted with a high-sensitivity real-time CCD camera.

\section{Western Blotting}

Overnight bacterial cultures were diluted 1:100 to optical density 1.0 at $600 \mathrm{~nm}$. Whole-cell lysates were resolved on $12 \%$ sodium dodecyl sulfate-polyacrylamide gels, and transferred to polyvinylidene difluoride membranes. Subsequently, membranes were probed with a 1:10,000 dilution of antibodies to flagellin (Abcam: 93713) or DnaK (Abcam: ab69617), followed by a 1:2,000 dilution of goat anti-rabbit (Abcam: ab6721) or anti-mouse immunoglobulin G (Abcam: ab205719) conjugated to horseradish peroxidase. Blots were visualized on a chemiluminescence detection system following reaction with ECL enhanced chemiluminescence reagent. Proteins were quantified using Amersham Imager 600 software (GE Healthcare).

\section{Electrophoretic Mobility Shift Assay}

GmrA N-terminally tagged with $6 \times$ His was expressed in E. coli BL21, using the expression vector pET28a and purified from soluble extracts using nickel columns (GE Healthcare: 17057501). Protein concentration was determined by Bradford assay, and stored in aliquots at $-70^{\circ} \mathrm{C}$. PCR fragments encompassing regulatory regions of fliA (495 bp, -412 to +83 ) and $f \operatorname{lh} D(541 \mathrm{bp},-336$ to +205$)$, with respect to the corresponding transcriptional start sites, were amplified using genomic DNA of E. coli O157:H7 EDL933 as template (for the regulatory regions of fliA and $f h D$, see Salgado et al., 2013). A rpoS fragment (384 bp, +1052 to +1435 relative to the transcriptional start site) was also amplified, and used as negative control. The DNA fragments were then gel-purified and labeled with DIG using terminal transferase. Eectrophoretic mobility shift assays were performed using DIG Gel Shift Kit, $2^{\text {nd }}$ Generation (Roche: 03353591910) according to the manufacturer's instructions. Briefly, labeled DNA fragments $(1 \mathrm{nM})$ were incubated at $37^{\circ} \mathrm{C}$ for 20 min with various concentrations of purified GmrA His6 $(0-120 \mathrm{nM})$, in $20 \mu \mathrm{L}$ reactions containing band-shift buffer $(20 \mathrm{mM}$ Tris- $\mathrm{HCl} \mathrm{pH} \quad 7.5,80 \mathrm{mM} \mathrm{NaCl}, 0.1 \mathrm{mM}$ EDTA, and $1 \mathrm{mM}$ DTT). For competition assays, various concentrations of unlabeled DNA fragments $(10-150 \mathrm{nM})$ were added. Samples were separated by $10 \%$ native polyacrylamide gel electrophoresis, and transferred to nylon membranes. Labeled fragments were visualized on a chemiluminescence detection system following an enzyme immunoassay using anti-digoxigenin-AP, Fab-fragments, and the chemiluminescent substrate CSPD.

\section{Chromatin Immunoprecipitation- Quantitative PCR (ChIP-qPCR)}

Chromatin immunoprecipitation was performed as previously described (Lucchini et al., 2006; Davies et al., 2011) with some modification. Briefly, an inducible expression vector (pTRC99a) carrying $3 \times$ FLAG-tagged $g m r A$ was constructed and transformed into $\triangle g m r A$ mutant. Bacterial cultures were grown to mid-logarithmic phase until optical density 0.4 at $600 \mathrm{~nm}$, and protein expression was induced with $1 \mathrm{mM}$ IPTG for $30 \mathrm{~min}$ at $37^{\circ} \mathrm{C}$. To crosslink protein to DNA, formaldehyde was added to cultures to a final concentration of $1 \%$, and the mixture incubated at room temperature for $25 \mathrm{~min}$. Cross-linking was quenched by adding glycine at a final concentrations of $0.5 \mathrm{M}$. Cross-linked cells were then washed three times with ice-cold TBS, and sonicated extensively to generate DNA fragments of average size $\sim 500 \mathrm{bp}$. Cell debris was removed, and the resulting supernatant was used as cell extract for immunoprecipitation. Protein-DNA complexes were enriched with $3 \times$ FLAG antibody (Sigma: F1804) and protein A magnetic beads (Invitrogen: 10002D), following the manufacturer's instructions. As negative control, chromatin immunoprecipitation was performed using different aliquot without addition of antibodies. RNA were removed by incubation with RNaseA for $2 \mathrm{~h}$ at $37^{\circ} \mathrm{C}$, and proteins were removed by incubation with proteinase $\mathrm{K}$ for $2 \mathrm{~h}$ at $55^{\circ} \mathrm{C}$. The DNA sample was then purified using a PCR purification kit (Qiagen: 28104). To measure enrichment of fliA and $f$ lhDC promoters in immunoprecipitated DNA samples, relative-abundance quantitative PCR (qPCR) was performed with SYBR green mix. Relative enrichment was calculated by the $\Delta \Delta \mathrm{Ct}$ method (Livak and Schmittgen, 2001). Results shown represent average enrichment for three biological replicates. 


\section{Bioinformatics}

Orthologous groups were identified using OrthoFinder (Emms and Kelly, 2015), by which all nucleotide sequences were compared using a BLASTN all-against-all search with an E-value cutoff of $<10^{-4}$. Nucleotide sequences used to construct the phylogenetic tree were aligned in MAFFT (Katoh and Standley, 2013), and a maximum likelihood tree was constructed in PhyML (Guindon and Gascuel, 2003) based on the GTR model of nucleotide substitution with c-distributed rates among sites.

\section{RESULTS}

\section{OI-29 Is Not Required for 0157:H7 Adherence and LEE Gene Expression}

Previously, we showed by comparative transcriptomics that genes in OI-29 are significantly downregulated $3 \mathrm{~h}$ after incubation of E. coli O157:H7 with HeLa cells (Yang et al., 2015). We have now confirmed this result by qRT-PCR (Supplementary Table S3). To further investigate whether OI-29 is associated with virulence, we constructed a $\Delta$ OI-29 mutant and assessed its ability to adhere to host epithelial cells. This mutant was found to similarly adhere to HeLa cells (Figure 1A) and Caco-2 intestinal epithelial cells (Figure $1 \mathrm{~B}$ ) as the parental strain, while $\Delta e s c C$, a mutant of LEE genes and used as positive control, adhered at much

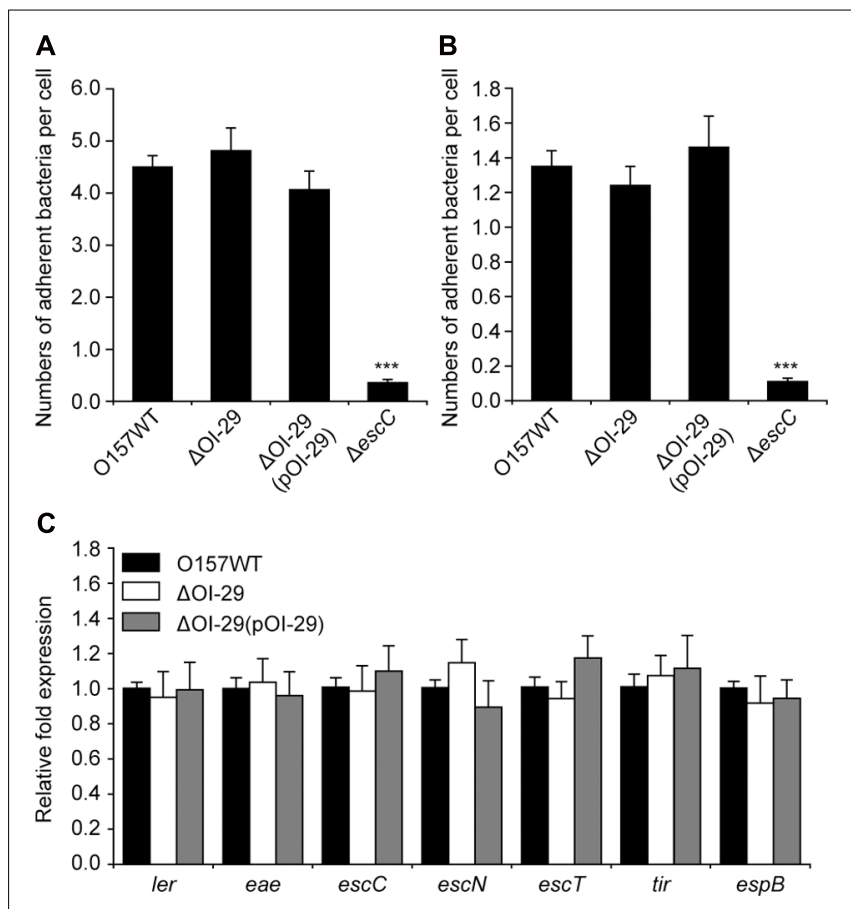

FIGURE 1 | Effect of OI-29 on Escherichia coli O157:H7 adherence and LEE gene expression. (A,B) Adhered bacteria were quantified $3 \mathrm{~h}$ after co-incubating $E$. coli O157: $\mathrm{H} 7$ wild-type, $\Delta \mathrm{Ol}-29$ mutant, $\Delta \mathrm{OI}-29$ complemented strain, and $\triangle$ escC (positive control) with HeLa (A) or Caco-2 cells (B). (C) E. coli strains were also grown to exponential phase, and analyzed by qRT-PCR for the expression of LEE genes, using 16S rRNA as internal control. Data are mean $\pm \mathrm{SD}, n=3 .{ }^{* * *} P \leq 0.001$ by Student's $t$-test. lower levels (Figures 1A,B). Accordingly, transcripts of seven representative LEE genes (ler, escT, escC, escN, eae, tir, and espB) were similarly abundant between parental and $\Delta$ OI-29 strains (Figure 1C). These results suggest that OI-29 is not required for E. coli $\mathrm{O} 157: \mathrm{H} 7$ adherence and LEE gene expression.

\section{Deletion of OI-29 Reduces Motility and Flagellar Biosynthesis in 0157:H7}

Motility was repressed in the $\Delta$ OI-29 mutant compared with that in wild-type E. coli O157:H7, with growth radius after $10 \mathrm{~h}$ at $30^{\circ} \mathrm{C}$ on motility agar 1.86 -fold smaller in the former than in the latter (Figures 2A,B). The motility defect was rescued by complementation with a low-copy plasmid (pACYC184) encoding OI-29 (Figures 2A,B). The motility of $\Delta \mathrm{OI}-29$ was not affected by introducing an empty pACYC184 into the mutant (Supplementary Figures S1A,B). The decreased motility of $\Delta \mathrm{OI}-$ 29 was also detected when the bacterium was grown at $37^{\circ} \mathrm{C}$ (Supplementary Figure S2). Of note, wild-type, $\Delta \mathrm{OI}-29$, and the complemented strain grew at similar rates in LB medium (Figure 2C), indicating that the decreased motility in $\Delta \mathrm{OI}-29$ was not due to slower growth. Strikingly, electron microscopy revealed that approximately $85 \%$ of wild-type and complemented cells $(n=500$ cells per strain) possessed 1-3 flagella, while approximately $80 \%$ of $\Delta$ OI- 29 cells $(n=500)$ were aflagellar. Representative transmission electron micrographs were shown in Figure 2D. These results suggested that loss of OI-29 represses flagellar biosynthesis.

Hence, expression of the flagellar genes $\mathrm{fliC}$ (encoding the major flagellin) and motA (encoding the flagella motor) was analyzed by qRT-PCR in both the parental strain and the $\Delta$ OI-29 mutant. Deletion of OI-29 was found to decrease the abundance of $f l i C$ and $m o t A$ transcripts by fourfold and threefold, respectively (Figure 2E). The transcription of flagellar genes in $\Delta$ OI-29 was not affected by introducing an empty pACYC184 into the mutant (Supplementary Figure S1C). Western blotting also revealed that FliC expression was significantly decreased in the mutant (Figure 2F). Transcription and translation were restored to wild-type levels upon complementation with a functional copy of $\Delta$ OI-29 (Figures 2E,F), confirming that OI29 enhances E. coli O157:H7 motility by upregulating flagellar synthesis.

\section{GmrA Is an Activator of Motility and Flagellar Biosynthesis in 0157:H7}

OI-29 in E. coli $\mathrm{O} 157: \mathrm{H7}$ is a 2,643-bp island that contains $z 0638, z 0639$ (named $g m r A$ ), and $z 0640$, which encode hypothetical proteins of unknown function (Supplementary Figure S3A). Notably, the gmrA gene product contains a putative DNA-binding domain (Supplementary Figure S3B), and therefore may act as a transcriptional regulator. Accordingly, the growth radius of $\Delta \mathrm{OI}-29$ on motility plates was restored to wild-type levels when complemented with gmrA but not with $z 0638$ and $z 0640$ (Supplementary Figure S4), suggesting that the motility defect is due only to $g m r A$. A $\Delta g m r A$ mutant was also constructed, and was found to have significantly reduced motility on semi-solid LB agar and flagellar biosynthesis, as 
A

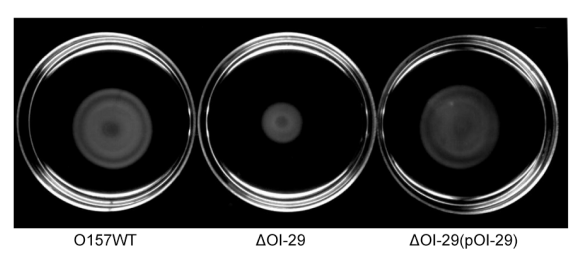

D

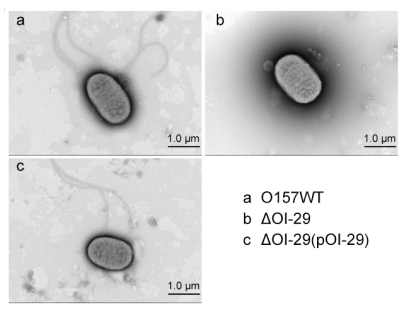

E

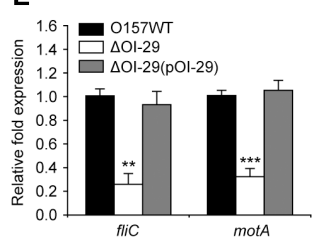

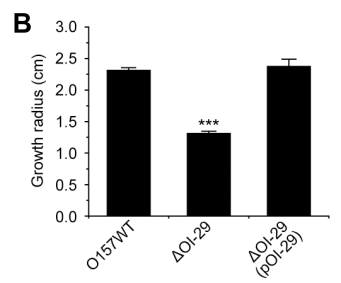

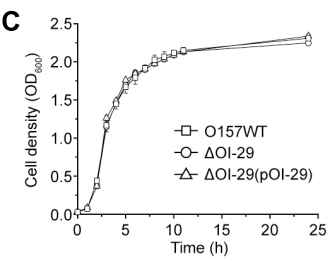

$\mathbf{F}$

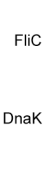

Flic
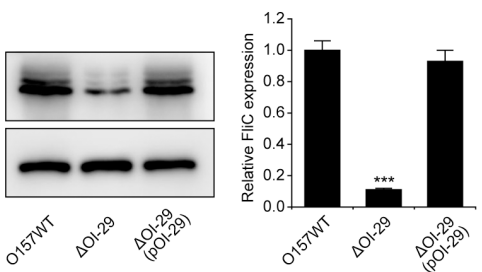

FIGURE 2 | Effect of Ol-29 on E. coli O157:H7 motility. Representative images of swimming motility (A), growth radius after $10 \mathrm{~h}$ at $30^{\circ} \mathrm{C}$ on motility agar (B), growth in LB medium (C), and representative transmission electron micrographs (D; scale bar, $1 \mu \mathrm{m}$ ) of E. coli O157: H7 wild-type, $\Delta$ OI-29 mutant, and complemented strain. The average number of flagella per cell, as estimated from 500 cells per strain, is 1.21 for wild-type, 0.25 for $\Delta$ Ol-29 mutant, and 1.37 for the complemented strain. (E) Strains grown to exponential phase were analyzed by qRT-PCR for fliC and motA, using $16 \mathrm{~S}$ rRNA as internal control. (F) Strains were also analyzed by immunoblotting for FliC, using DnaK as loading control. Bands were quantified by densitometry and normalized to DnaK. (B,E,F) Data are mean \pm SD, $n=3$.

${ }^{* *} P \leq 0.01 ;{ }^{* * *} P \leq 0.001$ by Student's $t$-test.

well as suppressed transcriptional and translational expression of flagellar genes in comparison to wild-type (Supplementary Figures S1D, S5). These defects were comparable to those in $\Delta$ OI-29, and were restored to wild-type levels when complemented with a low-copy plasmid carrying gmrA (Supplementary Figure S5). On the other hand, the growth radius and transcriptional expression of $f l i C$ and motA were not affected by deletion of $z 0638$ and $z 0640$ (Supplementary Figure S6), implying that these genes do not regulate bacterial motility. Collectively, these results confirm that GmrA is an activator of motility and flagellar synthesis in E. coli O157:H7.

\section{GmrA Activates Expression of Flagellar Genes via fliA}

As expression of flagellar genes is directly controlled by FlhDC and FliA, we tested whether GmrA interacts with either or both. We found that fliA transcripts were significantly less abundant in the $\triangle g m r A$ mutant, a defect rescued by a low-copy plasmid carrying gmrA (Figure 3A). In contrast, expression of $f h D$ and $f l h C$ was comparable among E. coli O157:H7 wild-type, $\triangle g m r A$, and complemented strain (Figure 3A). Electrophoretic mobility shift and competition assays suggested that GmrA binds specifically to the fliA promoter in vitro, but not to the flhDC promoter and rpoS (negative control) (Figure 3B). ChIP-qPCR also showed that the fliA promoter was enriched 7.75-fold in GmrA-ChIP samples than in mock-ChIP control samples, confirming that GmrA binds to the fliA promoter in vivo (Figure 3C). However, flhDC promoter and rpoS were not enriched in GmrA-ChIP samples (Figure 3C). These results suggest that GmrA binds directly and specifically to the fliA promoter to upregulate transcription.

Motility and expression of $f l i C$ and motA were significantly reduced in the $\Delta f l i A$ mutant compared with that in wild-type
(Figures 3D-F), confirming that FliA is a positive regulator of bacterial motility and flagellar synthesis. We also found that the growth radius and abundance of $f l i C$ and $m o t A$ transcripts were comparable between $\triangle f l i A$ and the double mutant $\triangle f l i A \Delta g m r A$ (Figures 3D-F), viz., deletion of gmrA has no impact on bacterial motility and flagellar gene expression in the $\Delta f l i A$ background. In addition, both bacterial motility and flagellar gene expression were restored to wild-type levels when an inducible plasmid carrying $f l i A$ was introduced into the $\triangle g m r A$ mutant (Figures 3D-F). Collectively, the data indicate that GmrA regulates E. coli O157:H7 motility and flagella synthesis through fliA.

\section{Optimal Conditions for gmrA Expression Are Similar to Those in the Human Intestine}

Expression of gmrA in E. coli O157:H7 grown to exponential phase or stationary phase in LB or DMEM (the tissue culture medium used for adherence assays) was examined by qRT-PCR. While the transcript level of gmrA was higher in LB-grown than the level in DMEM-grown E. coli O157:H7 under the same growth stage (Figure 4A), gmrA expressed higher in exponential phase than stationary phase E. coli O157:H7 grown in either medium (Figure 4A). To determine optimal conditions for gmrA expression, E. coli O157:H7 was grown in LB or DMEM at different temperatures, $\mathrm{pH}$ and osmolarity to exponential phase for qRT-PCR analysis. In either LB or DMEM, the optimal temperature for $g m r A$ expression is $37^{\circ} \mathrm{C}$, with transcript levels slightly reduced at 33 or $39^{\circ} \mathrm{C}$, but significantly diminished at 30 or $42^{\circ} \mathrm{C}$ (Figure $4 \mathbf{B}$ ). Buffering the medium at $\mathrm{pH}$ 7.0 elicited the highest levels of expression, while an increase or decrease in $\mathrm{pH}$ greatly reduced transcripts (Figure 4C). 


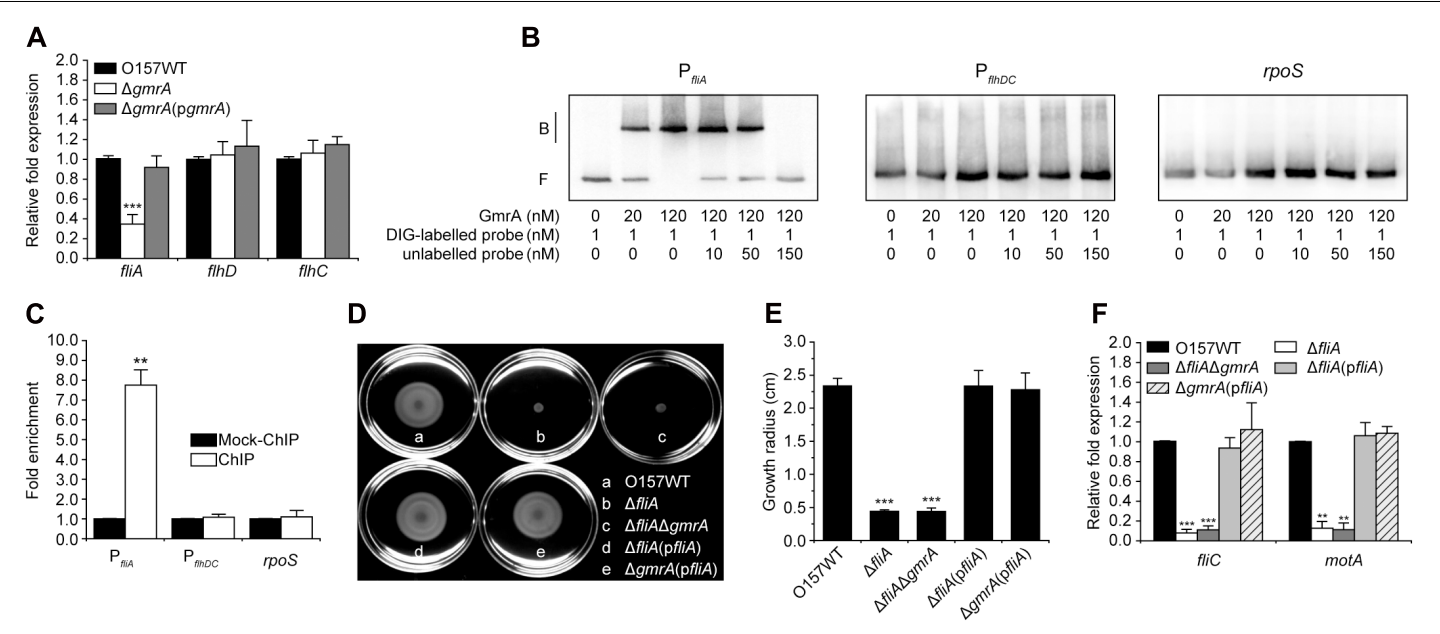

FIGURE 3 | GmrA regulates E. coli O157:H7 motility and flagellar synthesis through fliA. (A) Expression of fliA, flhD, and flhC in E. coli O157:H7 wild-type, $\triangle$ gmrA mutant, and complemented strain, as measured by qRT-PCR of cells in exponential phase, using 16S rRNA as internal control. (B) Electrophoretic mobility shift and competition assays of GmrA against the fliA and flhDC promoters, as well as rpos (negative control), with bound and free fragments marked B and F, respectively, and concentrations indicated at the bottom of each lane. Full blots are shown in Supplementary Figure S11. (C) Fold enrichment of fliA and flhDC promoters in GmrA-ChIP samples, as measured by ChIP-qPCR using rpoS as negative control. Representative images of swimming motility (D) and growth radius after $10 \mathrm{~h}$ at $30^{\circ} \mathrm{C}$ on motility agar (E) of $E$. coli $\mathrm{O} 157: \mathrm{H} 7$ wild-type, $\Delta$ fliA mutant, $\Delta$ fliA $\Delta$ gmrA double mutant, and corresponding complemented strains. (F) qRT-PCR for fliC and motA in cells grown to exponential phase, using $16 \mathrm{~S}$ rRNA gene as internal control. (A,C,E,F) Data are mean $\pm \mathrm{SD}, n=3$. ** $P \leq 0.01$; *** $P \leq 0.001$ by Student's t-test.

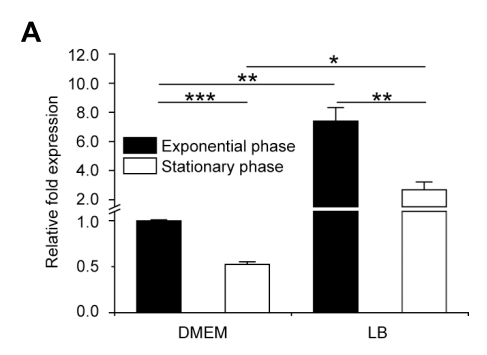

D

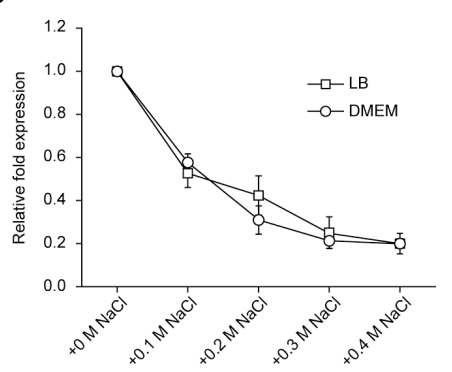

B

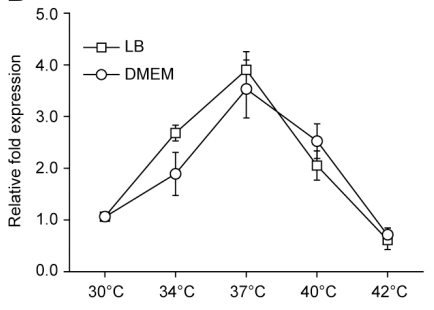

E

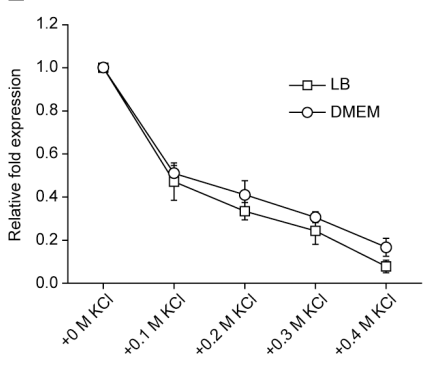

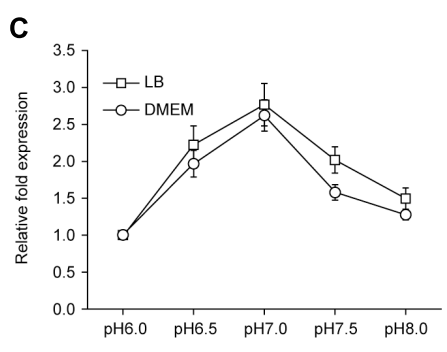

F

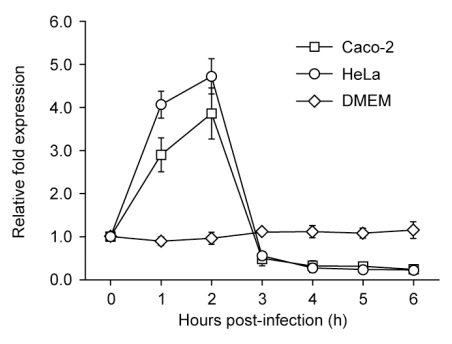

FIGURE 4 | Optimal conditions for gmrA expression. (A) qRT-PCR for gmrA in exponential-phased and stationary-phased E. coli O157:H7 grown in LB or DMEM medium. qRT-PCR for gmrA in exponential-phased E. coli O157:H7 grown in LB or DMEM medium at different temperatures (B), pH (C), NaCl concentrations (D), and $\mathrm{KCl}$ concentrations (E). (F) qRT-PCR of gmrA in E. coli O157:H7 incubated in DMEM alone or co-incubated with Caco-2 or HeLa epithelial cells for 1 to 6 h. Data are mean $\pm \mathrm{SD}, n=3$. ${ }^{*} P \leq 0.05 ; * * P \leq 0.01 ; * * P \leq 0.001$ by Student's $t$-test.

Expression was also maximal at physiological osmolarity, such that increased osmolarity from $\mathrm{NaCl}$ or $\mathrm{KCl}$ also significantly reduced transcription (Figures $4 \mathrm{D}, \mathrm{E}$ ). These results suggest that gmrA expression is maximal in conditions similar to those in the intestinal tract. However, $g m r A$ expression was not affected by the presence of bile salts and sodium bicarbonate, which are predominantly found in small intestine and common signals for virulence gene regulation (Supplementary Figure S7). Remarkably, expression was strongly induced in the first $2 \mathrm{~h}$ of co-incubating E. coli O157:H7 with Caco-2 or HeLa epithelial cells, but was significantly repressed $3 \mathrm{~h}$ post-infection (Figure 4F), indicating that GmrA regulates motility and flagella synthesis mainly at the initial phase of infection. The expression of $g m r A$ was at similar levels during the course of 
$6 \mathrm{~h}$ growth in DMEM in the absence of host epithelial cells (Figure 4F), indicating the induction of $g m r A$ in the first $2 \mathrm{~h}$ and repression $3 \mathrm{~h}$ onwards is dependent on the presence of host cells.

\section{GmrA Is a Widespread Regulator of Bacterial Motility in Pathogenic E. coli}

Bioinformatics analysis of 231 available E. coli genome sequence showed that OI-29 and gmrA are highly conserved and widely distributed in various E. coli lineages. Phylogenetic analysis also revealed that $E$. coli with OI-29 fall predominantly into four distinct clades, consisting of all pathogenic strains with only one exception (64 in total) (Supplementary Figure S8 and Supplementary Table S4). Clade 1 contains enteropathogenic E. coli $\mathrm{O} 55: \mathrm{H} 7$ and EHEC strains $\mathrm{O} 157: \mathrm{H} 7$ and $\mathrm{O} 145: \mathrm{H} 28$. Other three clades contain neonatal meningitis-associated E. coli strains CE10, IHE3034, RS218, and S88; uropathogenic E. coli strains IAI39, MS6198, and PMV-1; extraintestinal pathogenic E. coli strains UTI89, UMN026, PCN033, and PPECC42; avian pathogenic E. coli strains O1 and IMT5155; enteroaggregative E. coli 042 ; adherent invasive E. coli UM146; and other clinical isolate E. coli strains (Supplementary Figure S8 and Supplementary Table S4). To investigate whether orthologous gmrA genes also regulate motility, we deleted such genes from eight representative strains, consisting of two strains each of O157:H7 and O55:H7 and one strain each of O127:H6, avian $\mathrm{O} 2$ and $\mathrm{O} 2: \mathrm{H} 8$, and neonatal meningitis-associated O18. The growth radius on motility plates and expression of $f l i C$ in these mutants were significantly decreased compared with the corresponding wild-type strains (Figure 5), confirming that GmrA is a widespread regulator of E. coli motility and flagellar synthesis.

\section{DISCUSSION}

In this study, we report that OI-29 is required for the motility of O157. This is the third motility associated OI identified in O157, indicating the importance of motility control for the evolving of this pathogen, through specifically acquired regulators. In contrast to previously reported OI-1 that encodes a fliA repressor (Z0021), and OI-172 that encodes the putative DEAH box RNA helicase (Z5898) to promote flagella-based motility via $\mathrm{fliC}$, independent of fliA (Allison et al., 2012; Xu et al., 2013), OI-29 encoded GmrA promotes the fliA transcription, and thereby promotes flagellar synthesis. The regulation of fliA transcription by GmrA could be either direct or indirect, and this will be the subject of future studies. Our work significantly expands our understanding of bacterial motility control by providing a new example of fliA-dependent regulation, and increases the complexity of the regulatory network that governs flagellar genes. However, the two other OI-29 genes, z0638 and $z 0640$, have no obvious impact on O157:H7 motility, but may be involved in other bacterial processes, and thus require further characterization.

Bacterial flagellum is strictly regulated to prevent expression until environmental conditions are optimal, in order to avoid
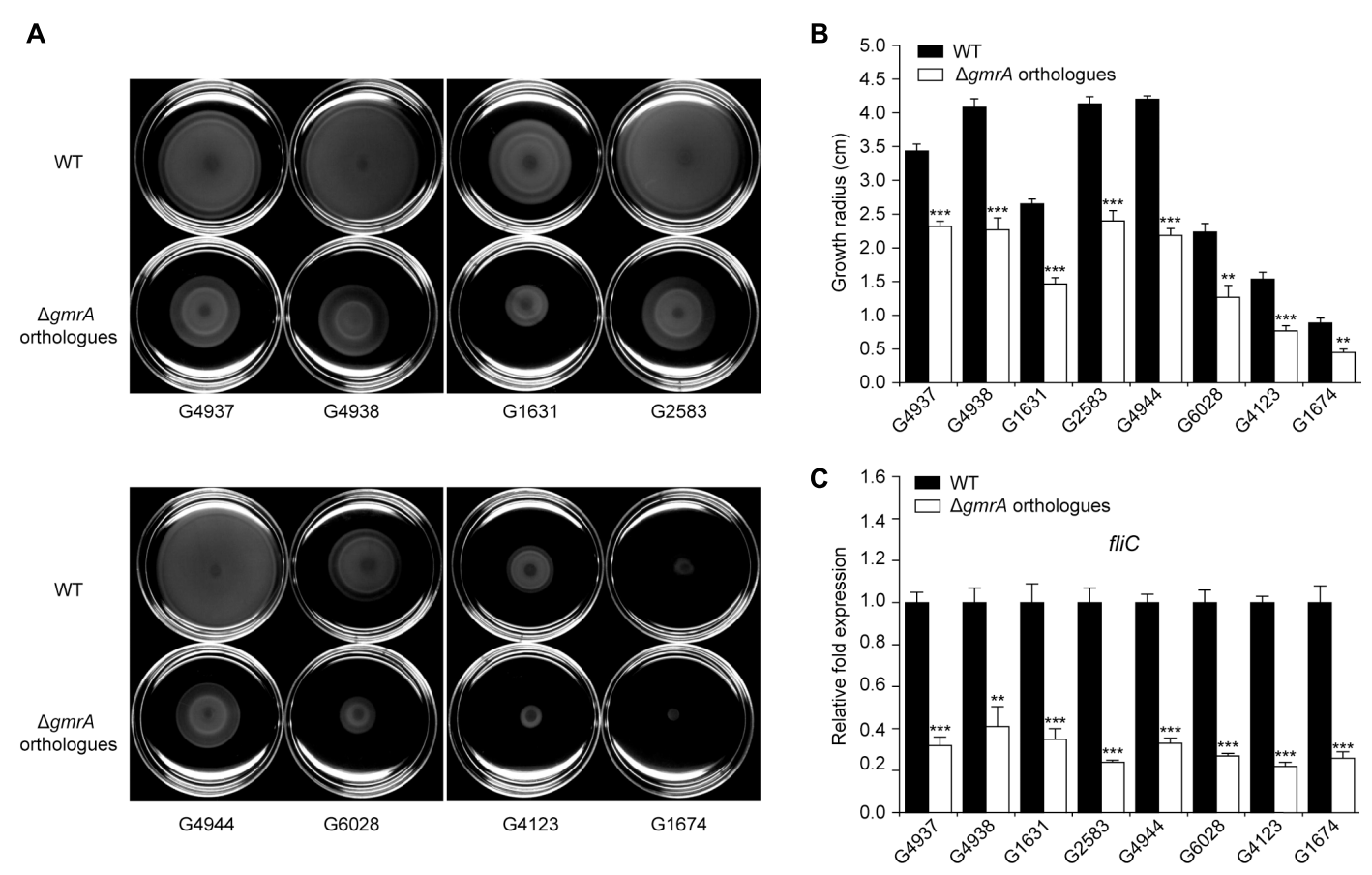

FIGURE $\mathbf{5}$ | GmrA is a widespread regulator of bacterial motility and flagella synthesis. Representative images of swimming motility (A) and growth radius after $10 \mathrm{~h}$ at $30^{\circ} \mathrm{C}$ on motility agar (B) of various $E$. coli strains and corresponding mutants from which orthologous gmrA were deleted. (C) qRT-PCR for fliC in various strains grown to exponential phase, using $16 \mathrm{~S}$ rRNA as internal control. (B,C) Data are mean $\pm \mathrm{SD}, n=3$. ${ }^{* *} P \leq 0.01$; ${ }^{* * *} P \leq 0.001$ by Student's $t$-test. 
high metabolic cost and ensure survival in different environments (Allison et al., 2012). We found that transcription of $g m r A$ was maximal at $37^{\circ} \mathrm{C}, \mathrm{pH} 7.0$, and physiological osmolarity, conditions that resemble those of the human intestinal tract. In addition, $g m r A$ expression significantly increased in the first $2 \mathrm{~h}$ of co-incubating E. coli O157:H7 with Caco-2 intestinal cells, but diminished almost completely from $3 \mathrm{~h}$ onwards. Therefore, we appear to have identified a mechanism driving a previously reported phenomenon, in which flagella are liberally formed by E. coli O157:H7 in early stages of infection, but are subsequently lost (Mahajan et al., 2009). Accordingly, we propose that during early infection, E. coli O157:H7 upregulates $g m r A$ in response to environmental changes in temperature, $\mathrm{pH}$, osmolarity, and presence of host cells, ultimately activating the expression of flagellar genes through fliA. The resulting increase in motility then enables the pathogen to reach and adhere to colonization sites in the host. After successful infection, motility becomes less critical, and cells then downregulate gmrA to inhibit flagellar synthesis, not only to save energy, but also to minimize host immunity, since bacterial flagellin is a potent antigen that elicits secretion of proinflammatory chemokines in human intestinal epithelial cells (Berin et al., 2002; Miyamoto et al., 2006). Nevertheless, further studies are required to reveal the precise regulatory impact of GmrA in flagellar synthesis and pathogenesis in vivo. For example, how exactly GmrA-activated flagellar synthesis contributes to virulence, and whether other environmental cues in the human intestine elicit flagellar synthesis remains to be established, as are the mechanisms for regulating GmrA expression under host conditions.

The abilities to induce attaching and effacing lesions and to produce Shiga toxins are considered the two most important virulence determinants in E. coli O157:H7 (Monteiro et al., 2016). While GmrA has no effect on the expression of LEE genes that are responsible for forming such lesions, we further examined the expression of two representative Shiga toxin genes (stx $1 A$ and $s t x 2 A)$ in wild-type, $\Delta \mathrm{OI}-29$, and complemented strains. Results show that expression of both genes was not affected by deletion of OI-29 in E. coli O157:H7 (Supplementary Figure S9). Therefore, GmrA contributes to O157:H7 virulence by affecting motility while having no effects on lesion formation and Shiga toxin production.

OI-29 is widespread in different pathotypes of E. coli strains, and these strains cluster predominantly into four distinct clades. Hence, OI-29 was likely gained through four independent evolutionary events. For example, the most recent common ancestor of $\mathrm{O} 55: \mathrm{H} 7, \mathrm{O} 157: \mathrm{H} 7$, and $\mathrm{O} 145: \mathrm{H} 28$ in clade 1 seems to have acquired OI-29 after diverging from E. coli O157:H16. In addition, almost all strains with OI-29 are important pathogens in human or animals, indicating that these hosts may have driven the acquisition of OI-29 during the evolution of those pathogenic strains.

The motility and the expression of flagella genes ( $f i A$ and $f l i C$ ) were also compared between E. coli O157:H7 and commensal E. coli K12. It was found that E. coli K12 was less motile on semi-solid LB agar than E. coli O157:H7 (Supplementary Figures S10A,B). In accordance, the transcriptional level of $f l i A$ and $f l i C$ was also lower in E. coli K12 (Supplementary
Figure S10C). Both capacities of E. coli K12 were largely enhanced when an expression plasmid carrying $g m r A$ was introduced into the strain (Supplementary Figure S10). These results suggest that $g m r A$ was acquired by pathogenic E. coli strains during evolution from commensal strains to enhance motility, which may provide advantages in survival and infection in vivo.

\section{CONCLUSION}

This study reveals a new example of regulators for the control of flagella synthesis. The laterally acquired GmrA is deployed by E. coli $\mathrm{O} 157: \mathrm{H} 7$ and likely many other pathogenic $E$. coli strains to enhance flagella synthesis and therefore motility during infection, highlighting the importance of motility in bacterial pathogenesis. The fact that GmrA is encoded in OI-29 demonstrates further that genomic islands contribute largely to bacterial pathogenesis by imparting new virulence traits, providing a rationale to investigate other uncharacterized OIs in E. coli O157:H7.

\section{AUTHOR CONTRIBUTIONS}

LF conceived and designed the experiments. BY, SW, JH, ZY, WH, LJ, and XL performed the experiments. BY and LF analyzed the data and wrote the paper.

\section{FUNDING}

This work was funded by the National Natural Science Foundation of China (NSFC) Key Program Grant (No. 31530083), the National Key Programs for Infectious Diseases of China (Nos. 2017ZX10303405-001 and 2017ZX10104002-001006), the National Special Project on Research and Development of Key Biosafety Technologies Grant (No. 2016YFC1200104); the NSFC General Program Grants 31470194, 31371259, and 81471904, the National Key Basic Research Program of China (No. 2016YFC1200100), the Natural Science Foundation of Tianjin (No. 17JCQNJC09300), the Fundamental Research Funds for the Central Universities (No. 63161105) and the International Science and Technology Cooperation Program of China (Nos. 2012DFG31680 and 2013DFR30640).

\section{ACKNOWLEDGMENTS}

We gratefully appreciate Dr. Dapeng Yan (Department of Immunology, School of Basic Medical Sciences, Fudan University, Shanghai, China) for providing the enteropathogenic E. coli O127:H6 E2348/69 strain.

\section{SUPPLEMENTARY MATERIAL}

The Supplementary Material for this article can be found online at: https://www.frontiersin.org/articles/10.3389/fmicb.2018. 00338/full\#supplementary-material 


\section{REFERENCES}

Allison, S. E., Silphaduang, U., Mascarenhas, M., Konczy, P., Quan, Q., Karmali, M., et al. (2012). Novel repressor of Escherichia coli O157:H7 motility encoded in the putative fimbrial cluster OI-1. J. Bacteriol. 194, 5343-5352. doi: 10.1128/JB.01025-12

Bavaro, M. F. (2012). E. coli O157:H7 and other toxigenic strains: the curse of global food distribution. Curr. Gastroenterol. Rep. 14, 317-323. doi: 10.1007/s11894012-02646

Berg, H. C. (2003). The rotary motor of bacterial flagella. Annu. Rev. Biochem. 72, 19-54. doi: 10.1146/annurev.biochem.72.121801.161737

Berin, M. C., Darfeuille-Michaud, A., Egan, L. J., Miyamoto, Y., and Kagnoff, M. F. (2002). Role of EHEC O157:H7 virulence factors in the activation of intestinal epithelial cell NF-kappaB and MAP kinase pathways and the upregulated expression of interleukin 8. Cell. Microbiol. 4, 635-648. doi: 10.1046/j.14625822.2002.00218.x

Caprioli, A., Morabito, S., Brugere, H., and Oswald, E. (2005). Enterohaemorrhagic Escherichia coli: emerging issues on virulence and modes of transmission. Vet. Res. 36, 289-311. doi: 10.1051/vetres:2005002

Chaban, B., Hughes, H. V., and Beeby, M. (2015). The flagellum in bacterial pathogens: for motility and a whole lot more. Semin. Cell Dev. Biol. 46, 91-103. doi: $10.1016 /$ j.semcdb.2015.10.032

Chevance, F. F., and Hughes, K. T. (2008). Coordinating assembly of a bacterial macromolecular machine. Nat. Rev. Microbiol. 6, 455-465. doi: 10.1038/ nrmicro1887

Claret, L., and Hughes, C. (2000). Functions of the subunits in the $\mathrm{FlhD}_{2} \mathrm{C}_{2}$ transcriptional master regulator of bacterial flagellum biogenesis and swarming. J. Mol. Biol. 303, 467-478. doi: 10.1006/jmbi.2000.4149

Connolly, J. P., Finlay, B. B., and Roe, A. J. (2015). From ingestion to colonization: the influence of the host environment on regulation of the LEE encoded type III secretion system in enterohaemorrhagic Escherichia coli. Front. Microbiol. 6:568. doi: 10.3389/fmicb.2015.00568

Datsenko, K. A., and Wanner, B. L. (2000). One-step inactivation of chromosomal genes in Escherichia coli K-12 using PCR products. Proc. Natl. Acad. Sci. U.S.A. 97, 6640-6645. doi: 10.1073/pnas.120163297

Davies, B. W., Bogard, R. W., and Mekalanos, J. J. (2011). Mapping the regulon of Vibrio cholerae ferric uptake regulator expands its known network of gene regulation. Proc. Natl. Acad. Sci. U.S.A. 108, 12467-12472. doi: 10.1073/pnas. 1107894108

Dibb-Fuller, M. P., Best, A., Stagg, D. A., Cooley, W. A., and Woodward, M. J. (2001). An in-vitro model for studying the interaction of Escherichia coli O157:H7 and other enteropathogens with bovine primary cell cultures. J. Med. Microbiol. 50, 759-769. doi: 10.1099/0022-1317-50-9-759

Duan, Q., Zhou, M., Zhu, L., and Zhu, G. (2013). Flagella and bacterial pathogenicity. J. Basic Microbiol. 53, 1-8. doi: 10.1002/jobm.201100335

Emms, D. M., and Kelly, S. (2015). OrthoFinder: solving fundamental biases in whole genome comparisons dramatically improves orthogroup inference accuracy. Genome Biol. 16:157. doi: 10.1186/s13059-015-0721-2

Erhardt, M., Namba, K., and Hughes, K. T. (2010). Bacterial nanomachines: the flagellum and type III injectisome. Cold Spring Harb. Perspect. Biol. 2:a000299. doi: $10.1101 /$ cshperspect.a000299

Flockhart, A. F., Tree, J. J., Xu, X., Karpiyevich, M., McAteer, S. P., Rosenblum, R., et al. (2012). Identification of a novel prophage regulator in Escherichia coli controlling the expression of type III secretion. Mol. Microbiol. 83, 208-223. doi: 10.1111/j.1365-2958.2011.07927.x

Gruenheid, S., Sekirov, I., Thomas, N. A., Deng, W., O’Donnell, P., Goode, D., et al. (2004). Identification and characterization of NleA, a non-LEE-encoded type III translocated virulence factor of enterohaemorrhagic Escherichia coli O157:H7. Mol. Microbiol. 51, 1233-1249. doi: 10.1046/j.1365-2958.2003. 03911.x

Guindon, S., and Gascuel, O. (2003). A simple, fast, and accurate algorithm to estimate large phylogenies by maximum likelihood. Syst. Biol. 52, 696-704. doi: 10.1080/10635150390235520

Hayashi, T., Makino, K., Ohnishi, M., Kurokawa, K., Ishii, K., Yokoyama, K., et al. (2001). Complete genome sequence of enterohemorrhagic Escherichia coli O157:H7 and genomic comparison with a laboratory strain K-12. DNA Res. 8, 11-22. doi: 10.1093/dnares/8.1.11
Ho, N. K., Henry, A. C., Johnson-Henry, K., and Sherman, P. M. (2013). Pathogenicity, host responses and implications for management of enterohemorrhagic Escherichia coli O157:H7 infection. Can. J. Gastroenterol. 27, 281-285. doi: 10.1155/2013/138673

Karmali, M. A., Mascarenhas, M., Shen, S., Ziebell, K., Johnson, S., Reid-Smith, R., et al. (2003). Association of genomic O island 122 of Escherichia coli EDL 933 with verocytotoxin-producing Escherichia coli seropathotypes that are linked to epidemic and/or serious disease. J. Clin. Microbiol. 41, 4930-4940. doi: 10.1128/ Jcm.41.11.4930-4940.2003

Katoh, K., and Standley, D. M. (2013). MAFFT multiple sequence alignment software version 7: improvements in performance and usability. Mol. Biol. Evol. 30, 772-780. doi: 10.1093/molbev/mst010

Livak, K. J., and Schmittgen, T. D. (2001). Analysis of relative gene expression data using real-time quantitative PCR and the $2^{-\Delta \Delta C_{\mathrm{T}}}$ method. Methods 25, 402-408. doi: 10.1006/meth.2001.1262

Lucchini, S., Rowley, G., Goldberg, M. D., Hurd, D., Harrison, M., and Hinton, J. C. (2006). H-NS mediates the silencing of laterally acquired genes in bacteria. PLoS Pathog. 2:e81. doi: 10.1371/journal.ppat.0020081

Mahajan, A., Currie, C. G., Mackie, S., Tree, J., McAteer, S., McKendrick, I., et al. (2009). An investigation of the expression and adhesin function of H7 flagella in the interaction of Escherichia coli O157 : H7 with bovine intestinal epithelium. Cell. Microbiol. 11, 121-137. doi: 10.1111/j.1462-5822.2008. 01244.x

McCarter, L. L. (2006). Regulation of flagella. Curr. Opin. Microbiol. 9, 180-186. doi: 10.1016/j.mib.2006.02.001

Mellies, J. L., Barron, A. M., and Carmona, A. M. (2007). Enteropathogenic and enterohemorrhagic Escherichia coli virulence gene regulation. Infect. Immun. 75, 4199-4210. doi: 10.1128/IAI.01927-06

Miyamoto, Y., Iimura, M., Kaper, J. B., Torres, A. G., and Kagnoff, M. F. (2006). Role of Shiga toxin versus H7 flagellin in enterohaemorrhagic Escherichia coli signalling of human colon epithelium in vivo. Cell. Microbiol. 8, 869-879. doi: 10.1111/j.1462-5822.2005.00673.x

Monteiro, R., Ageorges, V., Rojas-Lopez, M., Schmidt, H., Weiss, A., Bertin, Y., et al. (2016). A secretome view of colonisation factors in Shiga toxin-encoding Escherichia coli (STEC): from enterohaemorrhagic E. coli (EHEC) to related enteropathotypes. FEMS Microbiol. Lett. 363:fnw179. doi: 10.1093/femsle/ fnw179

Murphy, K. C., and Campellone, K. G. (2003). Lambda Red-mediated recombinogenic engineering of enterohemorrhagic and enteropathogenic E. coli. BMC Mol. Biol. 4:11. doi: 10.1186/1471-2199-4-11

Perna, N. T., Plunkett, G. III, Burland, V., Mau, B., Glasner, J. D., Rose, D. J., et al. (2001). Genome sequence of enterohaemorrhagic Escherichia coli O157:H7. Nature 409, 529-533. doi: 10.1038/35054089

Salgado, H., Peralta-Gil, M., Gama-Castro, S., Santos-Zavaleta, A., MunizRascado, L., Garcia-Sotelo, J. S., et al. (2013). RegulonDB v8.0: omics data sets, evolutionary conservation, regulatory phrases, cross-validated gold standards and more. Nucleic Acids Res. 41, D203-D213. doi: 10.1093/nar/gks 1201

Tasara, T., and Stephan, R. (2007). Evaluation of housekeeping genes in Listeria monocytogenes as potential internal control references for normalizing mRNA expression levels in stress adaptation models using real-time PCR. FEMS Microbiol. Lett. 269, 265-272. doi: 10.1111/j.1574-6968.2007. 00633.x

Tree, J. J., Roe, A. J., Flockhart, A., McAteer, S. P., Xu, X., Shaw, D., et al. (2011). Transcriptional regulators of the GAD acid stress island are carried by effector protein-encoding prophages and indirectly control type III secretion in enterohemorrhagic Escherichia coli O157:H7. Mol. Microbiol. 80, 1349-1365. doi: 10.1111/j.1365-2958.2011.07650.x

Watanabe, H. (2012). Pathogenesis of enterohaemorrhagic Escherichia coli infection. Nihon Rinsho 70, 1318-1322.

Wong, A. R., Pearson, J. S., Bright, M. D., Munera, D., Robinson, K. S., Lee, S. F., et al. (2011). Enteropathogenic and enterohaemorrhagic Escherichia coli: even more subversive elements. Mol. Microbiol. 80, 1420-1438. doi: 10.1111/j.13652958.2011.07661.x

Xu, Y., Xu, X., Lan, R., Xiong, Y., Ye, C., Ren, Z., et al. (2013). An O island 172 encoded RNA helicase regulates the motility of Escherichia coli O157:H7. PLoS One 8:e64211. doi: 10.1371/journal.pone.0064211 
Yang, B., Feng, L., Wang, F., and Wang, L. (2015). Enterohemorrhagic Escherichia coli senses low biotin status in the large intestine for colonization and infection. Nat. Commun. 6:6592. doi: 10.1038/ncomms7592

Yin, X., Chambers, J. R., Wheatcroft, R., Johnson, R. P., Zhu, J., Liu, B., et al (2009a). Adherence of Escherichia coli O157:H7 mutants in vitro and in ligated pig intestines. Appl. Environ. Microbiol. 75, 4975-4983. doi: 10.1128/AEM. 00297-09

Yin, X., Wheatcroft, R., Chambers, J. R., Liu, B., Zhu, J., and Gyles, C. L. (2009b). Contributions of $\mathrm{O}$ island 48 to adherence of enterohemorrhagic Escherichia coli $\mathrm{O} 157: \mathrm{H} 7$ to epithelial cells in vitro and in ligated pig ileal loops. Appl. Environ. Microbiol. 75, 5779-5786. doi: 10.1128/AEM.00507-09
Conflict of Interest Statement: The authors declare that the research was conducted in the absence of any commercial or financial relationships that could be construed as a potential conflict of interest.

Copyright (c) 2018 Yang, Wang, Huang, Yin, Jiang, Hou, Li and Feng. This is an open-access article distributed under the terms of the Creative Commons Attribution License (CC BY). The use, distribution or reproduction in other forums is permitted, provided the original author(s) and the copyright owner are credited and that the original publication in this journal is cited, in accordance with accepted academic practice. No use, distribution or reproduction is permitted which does not comply with these terms. 